\title{
MOTRICIDAD Y COGNICIÓN EN EL DÉFICIT DE ATENCIÓN E HIPERACTIVIDAD TDAH
}

\section{MOTOR SKILLS AND COGNITION IN ATTENTION DEFICIT HYPERACTIVITY DISORDER (ADHD)}

\author{
José Armando Vidarte Claros ${ }^{2}$ \\ Consuelo Vélez Alvarez ${ }^{3}$ \\ Oscar Hernán Moscoso Ariza ${ }^{4}$ \\ Francia Restrepo de Mejía
}

Palabras clave: Cognición, trastorno de la cognición del movimiento, déficit de atención con hiperactividad, parálisis de la motricidad, perfil psicomotor.

Keywords: : Cognition, Movement Disorder, Attention Deficit Hyperactivity Disorder, Motor Skills, Psychomotor Profile.

\section{RESUMEN}

Lo expuesto a continuación es una revisión sobre el trastorno por Déficit de la Atención e Hiperactividad el cual representa, entre otros, uno de los aspectos relevantes para la fundamentación teórica y el avance de la neurociencia cognitiva en este campo en particular, toda vez que los procesos de atención y memoria subyacen en la fisiopatología del sistema nervioso humano. El TDAH es una de las patologías crónicas más frecuentes en la infancia, se presenta su definición, epidemiología, diagnóstico y tratamiento, y el uso de las técnicas neurofisiológicas denominadas Potenciales relacionados a eventos cognitivos para la detección temprana y para mejorar el diagnóstico diferencial y los procesos de intervención, reduciendo así el impacto negativo que causa en nuestra sociedad.

\section{ABSTRACT}

This document consists of three steps. Firstly, some conceptual aspects about motor skills and cognition are stated. Secondly, the aspects about the disorders
1.Traducción: Blanca Estela Giraldo. Centro de Traducción del Instituto de Idiomas UAM

2. Ph.D en Ciencias de la Actividad Física y el deporte. Profesor Titular Universidad Autónoma de Manizales, líder del grupo de investigación Cuerpo Movimiento, UAM.

3. Ph.D en Salud Pública. Profesora Titular Universidad Autónoma de Manizales. Integrante del grupo de investigación cuerpo-Movimiento, UAM.

4 Ph.D en Neurociencia y B i o logía d e l comportamiento. Profesor Titular de la Universidad Autónoma de Manizales. Líder del grupo de i n v e s t i g a c i ó $n$ Neuroaprendizaje, UAM.

5. Ph.D en Ciencias Sociales Niñez y Juventud. Profesora Titular de la Universidad Autónoma de Manizales. Profesora Titular de la Universidad de Caldas. Coordinadora del Laboratorio de Neurofisiología, UAM

Recibido: febrero 15 de 2010 Aprobado: abril 20 de 2010

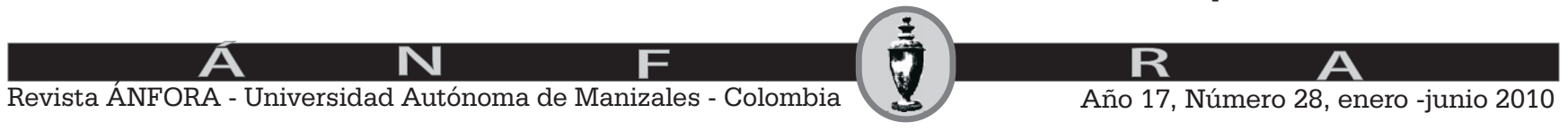


of the motor development and their relations with the cognitive processes are showed, specially dealing with Attention Deficit Hyperactivity Disorder (ADHD). Finally, some neurophysiological implications in psychomotor development disorders are developed and possible relations between the motor skills and ADHD are stated.

\section{Introducción}

Este documento se estructura en tres momentos: en el primero se establecen algunos aspectos conceptuales en torno a la motricidad y la cognición, en segundo lugar se muestran los aspectos sobre las alteraciones del desarrollo motriz y su relación con los procesos cognitivos, especialmente desde el abordaje del trastorno por déficit de atención e hiperactividad TDAH, y por último, se desarrollan algunas de las implicaciones neurofisiológicas en las alteraciones del desarrollo psicomotor y se plantean posibles relaciones entre la motricidad y el trastorno.

\section{Sobre el concepto de MOTRICIDAD}

La motricidad, es concebida como el campo del saber en el que se pone en juego la función del valor social, cultural, ético, político, cognitivo, imaginativocreativo, estético y motriz. La motricidad, entonces es un campo del saber cuyas fronteras las constituye el ser humano en movimiento (Parlebas, 2007, Trigo y Col 2003, Murcia, 2006, 2007, Hurtado, 2007). La característica general de esta definición de motricidad es que rompe con la consideración meramente funcional e instrumental del movimiento humano y se centra en el ser humano en movimiento. El cuerpo tiene sentido sólo en el contexto del sujeto corpóreo, lo que permite plantear entonces que la motricidad es la expresión de sentido de esa corporeidad.

La base de desarrollo de la motricidad la constituyen expresiones como lo artístico, la recreación, la actividad física, el deporte y la salud, también conocidas como ludomotricidad (recreación), la paidomotricidad (Educación Física), la ergomotricidad (Deporte). Lo anterior permite mostrar que no es posible la simplificación instrumental del movimiento, en términos de destrezas, habilidades o capacidades independientes de la vivencia; sino que estas categorías corresponden a toda la complejidad del ser humano en movimiento.

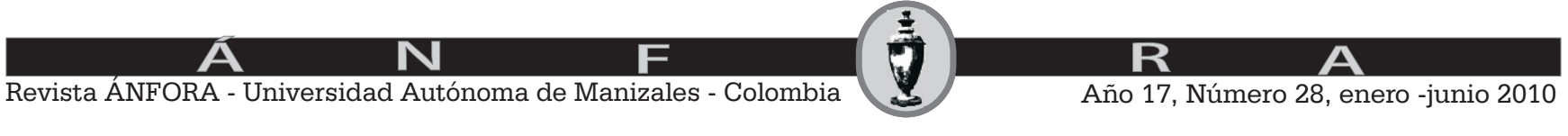


Los nuevos paradigmas consideran el movimiento como una subcategoría de la motricidad y, en consecuencia, es una de las manifestaciones de ésta, la cual se centra en un ser humano multidimensional y en un movimiento intencional que genera trascendencia. Sin embargo, desde la perspectiva de la corporeidad, por su complejidad, la motricidad desborda el concepto de movimiento. Esta visión sobre el concepto de movimiento (humano) es infinitamente más rica y más profunda que la visión mecanicista del movimiento ofrecida por la biofisiología fruto del paradigma cartesiano y de los conceptos newtonianos de la realidad. (Feitosa, 1993)

Por medio de la motricidad el cuerpo alcanza la corporeidad y a través de su energía expresa su capacidad de movimiento de tal forma que alcance la creatividad suficiente para generar la expresión y comunicación. Es a partir de la motricidad donde se descubre el cuerpo liberado de gastos energéticos inútiles. El lenguaje corporal como forma de expresión abarca diferentes planos siendo comunicación espontánea e instintiva; que acompaña toda expresión verbal; puede hacer una acción intencionada; es material informático, real y ficticio, y sus elementos fundamentales son el espacio, el tiempo y el cuerpo, es decir la unidad psicomotora.

La motricidad concebida como una vivencia de la corporeidad permite explicar las acciones que implican desarrollo de lo humano (Rey cao y otros, 1999), donde la corporeidad es la vivenciación del hacer, sentir, pensar, y querer de manera que se pueda identificar corporeidad con humanidad ya que el ser humano es y vive sólo a través de su corporeidad. En este sentido Trigo 1999, intenta diferenciar este término de la realidad física del cuerpo y del tiempo, destaca ciertos rasgos que pretende son exclusivos de la conducta humana pero que, en todo caso y con otras palabras, se refieren a nociones psicológicas tales como la conciencia, lo volitivo, lo cognitivo, lo afectivo o lo expresivo. Por tanto se asume que mientras el cuerpo solo hace, la corporeidad permite la existencia del ser humano mediante la unidad del hacer, el saber, el pensar, el sentir, el comunicar y el querer (pienso y siento al tiempo que hago; actúo porque siento y pienso).

Como dice Vítor da Fonseca en sus investigaciones sobre la evolución del ser humano: "la motricidad retrata, en términos de acción, los productos y los procesos funcionales creadores de nuevas acciones sobre acciones anteriores. Por la motricidad utilizadora, exploratoria, inventiva y constructiva, el Hombre y

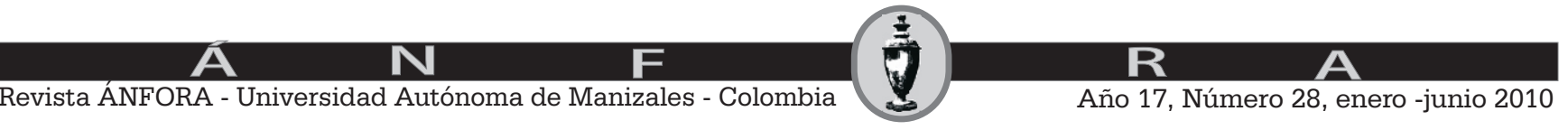


el niño, humanizando, esto es, socializando el movimiento, adquirirán el conocimiento" (Da Fonseca, 1989: 314-315).

Las teorías de este autor tienen múltiples influencias, pero entre ellas destacan las de Wallon (1970) con su base psicobiológica y las de Luria (1979) en su concepción neurobiológica. En sus trabajos, Da Fonseca afirma que la ontogénesis de la motricidad es el espejo de la filogénesis humana. El crecimiento, como aumento cuantitativo (estructura), y el desarrollo, como aumento cualitativo (complejidad), son manifestaciones del mismo fenómeno. “...Por ello se demuestra que los músculos (como órganos efectores por excelencia) son los instrumentos privilegiados por los cuales los seres humanos comunican sus pensamientos. El movimiento voluntario y el ajuste postural son las claves de la inteligencia y de la comunicación humana. El pensamiento es la culminación de la acción, ambos son las dos caras sublimes de la actividad psíquica superior. Así evolucionó la conciencia humana a nivel histórico (aspecto filogenético) y así se da la información de la inteligencia del niño (aspecto ontogenético)..." (Da Fonseca, 1988, p.47).

\section{Sobre el concepto de COGNICION}

Hablar de cognición permite remitir el concepto a la etimología latina de los términos conocimiento y conocer. El significado de la palabra conocer es "captar o tener la idea de una cosa, llegar a saber su naturaleza, cualidades y relaciones, mediante las facultades mentales". Para Neisser (1976), cualquier cosa que conozcamos acerca de la realidad, tiene que ser mediada, no sólo por los órganos de los sentidos, sino por un complejo de sistemas que interpretan y reinterpretan la información sensorial. El término cognición es definido como los procesos mediante los cuales el input sensorial es transformado, reducido, elaborado, almacenado, recobrado o utilizado. Los términos sensación, percepción, imaginación, recuerdo, solución de problemas, etc. se refieren a etapas o aspectos hipotéticos de la cognición. Neisser (1976) entiende por cognición: primero el conjunto de procesos mentales que tienen lugar entre la recepción de estímulos y la respuesta a éstos, y segundo las funciones complejas que operan sobre las representaciones perceptivas o recobradas de la memoria a largo plazo.

Corresponden a las estructuras mentales organizadoras que influyen en la interpretación de la información, influyendo en la configuración con la que se fija

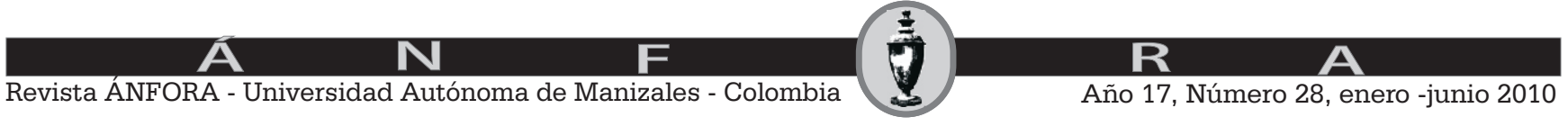


y evoca la información en la memoria de largo plazo determinando en parte la respuesta conductual. Son procesos estructurales inconscientes que derivan de experiencias del pasado, facilitan la interpretación de estímulos y afectan la dirección de conductas futuras, existiendo esquemas para distintas situaciones.

Los principales procesos cognitivos inherentes a la naturaleza humana maduran de manera ordenada en el desarrollo humano y las experiencias pueden acelerar o retardar el momento que estos hagan su aparición, llevando finalmente al complejo proceso denominado Aprendizaje. Es entonces necesario a partir de estos dos conceptos el de motricidad y el de cognición reconocer como su articulación permite el desempeño de manera coherente, adecuada y compleja del sujeto en cada uno de los contextos donde se desenvuelve. Por tanto cuando se presenta una alteración en cualquiera de los dos se verá afectado el otro y sus niveles de interacción.

A continuación se plantean algunos elementos que muestran como las alteraciones cognitivas desencadenan alteraciones motrices especialmente en el trastorno por déficit de atención e hiperactividad las cuales modifican el desempeño del sujeto en los contextos.

\section{ALTERACIONES DEL DESARROLLO MOTRIZY COGNICION}

En este apartado se hará el abordaje de las alteraciones motrices tomando como referencia sujetos que presentan trastornos de déficit de atención e hiperactividad (TDAH). Algunos estudios en los que se ha comparado la habilidad motriz fina de sujetos con TDAH con un grupo control, han encontrado que los primeros mostraban menor habilidad motriz que los segundos y que el tipo de dificultades observadas difería según los subtipos del trastorno (Piek, Pitcher y Hay, 1999; Steger, Imhof, Coutts, Gundelfinger, Steinhausen y Bradeis, 2001). En este sentido, los autores encontraron que los hombres afectados por déficit atencional y los que corresponden al tipo combinado (déficit atencional e hiperactividad) exhibían menor destreza que los adscritos al tipo impulsividadhiperactividad y que los del grupo control. Así mismo, en general, todos los niños con TDAH tenían peor desempeño de la motricidad fina que los controles. Estas deficiencias se traducen tanto en la torpeza para estar con su cuerpo como para ocupar el espacio y moverse en él con una movilidad intencional y simbolizada, suficientemente fluida (Mazet y Houzal, 1981). 
En el estudio de Steger et al. (2001) se matizó el tipo de alteración motriz que, específicamente, se evidenciaba en la rapidez de la respuesta y en la calidad y perfección de la ejecución de la tarea que, en todos los casos, era peor en los sujetos con TDAH que en los controles. Sin embargo, algunos estudios discrepan con estos resultados, señalando la ausencia de diferencias motrices entre las personas con TDAH y los controles (Leung y Connolly, 1998).

En general, se estima que más del $50 \%$ de los niños con TDAH pueden tener problemas motrices (Castroviejo, 2004; Piek et al., 1999; y Barckley, Du Paul y McMurray, 1990). Sin embargo, Kasdejö et al. (1998), Doyle, Wallen y Whitmont (1995) y Castroviejo (2004) señalan que el diagnóstico y seguimiento clínico de los pacientes (incluyendo la exploración neurológica) es, en su mayor parte, muy superficial. Estos autores explican que esta situación podría derivarse del hecho de que quienes realizan el diagnóstico y tratamiento suelen ser médicos y psicólogos que se centran, prioritariamente, en los síntomas de su ámbito de competencia y que, por lo general, desconocen los matices de la motricidad.

En cuanto a los neurólogos infantiles, los autores antes citados señalan que, aunque realizan exploración neurológica, no suele ser completa y, además, no reseñan las anomalías motrices que pudieran observarse. La escasa importancia concedida al desarrollo psicomotor, y más específicamente a la motricidad, dentro de los síntomas el TDAH, junto a la prevalencia de estas alteraciones en los pacientes afectados por este síndrome, constituyen una laguna en el conocimiento del TDAH. Superar este desconocimiento podría tener interés tanto desde el punto de vista teórico como aplicado.

Partiendo de características motrices predominantes en los niños con el TDAH, Bauermeister (2002) muestra la existencia de una proporción elevada de niños con dicho déficit que presentan dificultades en el desarrollo motor grueso. Como resultado de esas deficiencias, los sujetos pueden mostrarse torpes en sus movimientos, manifestando dificultad para correr y saltar. En lo que concierne al desarrollo motor fino, las dificultades se manifestarían en tareas que implican agarrar objetos (tales como el tenedor y el cuchillo), abotonarse la ropa, jugar con una bola, colorear dentro de los límites de la figura, escribir sobre líneas o en un tamaño uniforme, o ejecutar la escritura con una caligrafía aceptable y completar el trabajo escrito en el salón de clase.

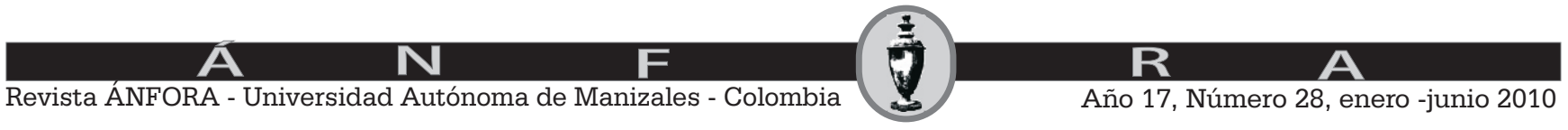


Por otra parte, Orjales (2002, citando a Zentall y Smith 1993) plantea que frecuentemente se ha asociado la hiperactividad infantil con problemas de coordinación motriz, y afirma que, en la actualidad, existen datos suficientes como para sostener que los niños hiperactivos tienen déficits visomotores, tiempos de reacción motriz mayores (necesitan más tiempo para dar respuesta motriz ante la aparición de un estímulo) y cometen mayor número de errores. Estos niños, además, suelen tener dificultades en actividades de psicomotricidad fina, son torpes para ensartar, modelar con plastilina, colorear de forma controlada, servir la leche sin derramarla, abrir bolsas y paquetes prensados, abrochar botones pequeños y hacer el lazo a los zapatos, tal como señalaba Bauermeister (2002).

Yochman, Ornoy y Parush (2006) publicaron un estudio cuyo objetivo era comparar el funcionamiento del desarrollo motor fino, motor grueso y visomotor de niños de 6 años; se trabajó 49 niños con TDAH y 48 niños normales, comparando sus ejecuciones. Los resultados mostraron la existencia de diferencias significativas entre ambos grupos, indicando que el desarrollo motor de los niños con TDAH era significativamente bajo con relación a los demás niños, en todas las medidas perceptivo-motrices. Además, mostraron la existencia de correlaciones significativas entre todas las variables motrices consideradas, con relación a los niños hiperactivos y desatentos. Asimismo, los análisis de regresión indicaron que el total de los resultados motores y visomotores eran predictores significativos de clasificación del grupo con y sin TDAH. Estos resultados confirman la importancia de la valoración temprana y el tratamiento del trastorno.

Otro estudio, sobre la influencia del Desorden de la coordinación de desarrollo y el Déficit de atención en los movimientos asociados de los niños, elaborado por Licari, Larkin y Miyahara (2006), tenía como objetivo determinar la relación entre los movimientos asociados (AMs) y el nivel de actuación motriz de los niños. Se investigó si los niños con el Desorden de Coordinación de Desarrollo (DCD), los que tenían TDAH y los sanos diferían en la severidad de los movimientos asociados. La muestra total fue de 10 niños con DCD, 10 con TDAH y 10 pertenecían a un grupo control. Tasando el desempeño en las tareas de AM., se encontró que los dos grupos con dificultades motrices tenían AMs significativamente más severos que el grupo sano. Estos resultados sugieren que el nivel de actuación motor debe ser considerado en investigaciones futuras, intentando entender las diferencias individuales en la severidad de

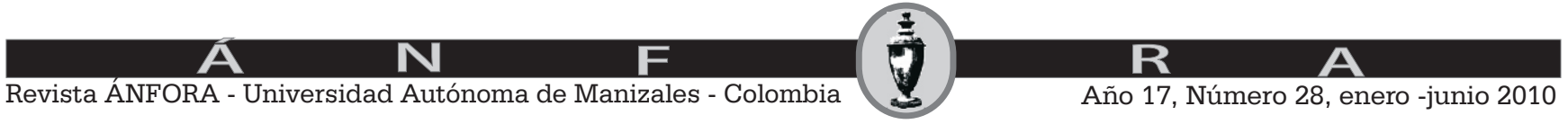


AMs, bien como una función del aprendizaje motor, bien como un déficit vinculado al TDAH.

Castroviejo (2004) realizó el estudio sobre las relaciones entre el TDAH y la capacidad para el deporte. La muestra estuvo constituida por 100 hombres con TDAH, de entre 6 y 16 años, con Cociente Intelectual normal, es decir, sobre el percentil 85. Se evaluó la motricidad a través de las opiniones de los padres respecto a la destreza de sus hijos para dibujar, escribir, y los problemas al andar, saltar y jugar. Los niños respondieron a un cuestionario de 8 preguntas relativas a su gusto por el deporte, el tipo de práctica deportiva, el puesto en el que jugaba si practicaba fútbol, la valoración de su desempeño deportivo (tanto personal como de padres y compañeros), la constancia y disciplina en la práctica deportiva y si se practicaba además, o en lugar de fútbol, otra actividad física.

Los resultados mostraron que la afectación neurológica más frecuente era la hipotonía. Ésta se manifestaba en todas las articulaciones en forma de hiperextensiones e hiperflexibilidad. En los pies, donde la hipotonía era más evidente, se manifestaba en pies plano-valgos, lo que obligaba a una marcha torpe. En general, la excesiva elasticidad de hombros y tronco coexistía con una importante potencia muscular global. El 67\% decían practicar deporte y de éstos un $20 \%$ elegían el fútbol, seguido del ciclismo con un $12 \%$. Natación, taekwondo, baloncesto, etc., resultaron de elección minoritaria. El 42\% de los que practicaban deporte manifestaron su motivación y constancia para realizar esta actividad, mientras que el 58\% reconocía que continuaban con el deporte a instancias de sus padres. Casi todos consideraban tener un desempeño deportivo satisfactorio, pero los padres de la mitad de ellos reconocían que su ejecución era deficiente en comparación con otros niños. En general, este estudio tiene el interés de indagar sobre las posibles relaciones entre la eficiencia motriz de los niños con TDAH y la práctica deportiva. Sin embargo, la evaluación de la motricidad, basada exclusivamente en las opiniones paternas y la del desempeño deportivo, medida sólo a través de las opiniones de los propios niños y de sus padres, está muy lejos de cumplir los requisitos metodológicos de una valoración motriz rigurosa.

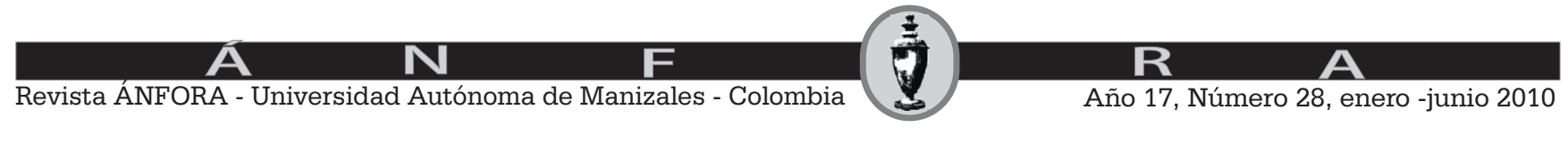




\section{IMPLICACIONES NEUROFISIOLÓGICAS EN LAS ALTERACIONES DEL DESARROLLO PSICOMOTOR DE LOS NIÑOS CON TDAH.}

En este epígrafe se plantearán algunos argumentos establecidos por diferentes autores en torno a las alteraciones presentes en el desarrollo psicomotor de los niños con TDAH. En concreto, se describirán las alteraciones correspondientes al lóbulo prefrontal, presentes en todas las manifestaciones del TDAH, y se propondrán algunos elementos que convergen en torno a las funciones ejecutivas, regidas por la actividad del córtex prefrontal y otras estructuras adyacentes a éste.

Las alteraciones de la función motriz parecen etiológicamente diferentes de las disfunciones atencionales, propias del TDAH. Sin embargo, parecen existir datos neurológicos y neuroquímicos que contribuyan a explicarlas.

Por una parte, la implicación del lóbulo frontal en el sistema general de autorregulación y, por otra, la deficiencia de la dopamina en la región prefrontal, parecen traducirse conductualmente en una incapacidad para controlar los impulsos, una dificultad para planificar y llevar a cabo una secuencia de acciones dirigidas a conseguir una meta y una actividad motriz excesiva, características conductuales del TDAH (Colby, 1991; Castellanos et al. 1994; 1996a; Barkley, et al. 1992).

Para comprender el TDAH sus manifestaciones y, especialmente, la posible vinculación entre las alteraciones cognitivas, conductuales y motrices, es necesario abordar los mecanismos subyacentes. En este sentido, cabe señalar que todas las manifestaciones del TDAH convergen en torno a las funciones ejecutivas, regidas por la actividad del córtex prefrontal y otras estructuras vinculadas a éste.

\section{Funciones del lóbulo prefrontal.}

La organización de la conducta y también de su dimensión motriz está relacionada con la actividad cortical y, especialmente, del lóbulo prefrontal, los ganglios basales y otras estructuras subcorticales. Los circuitos frontosubcorticales son los siguientes: un circuito motor, cuyo origen es el área motriz suplementaria, un circuito oculomotor, cuyo origen radica en los campos frontales oculares y tres circuitos que se originan en el córtex prefrontal.

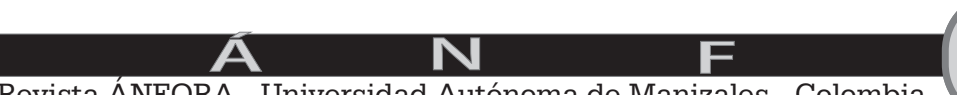


Estos cinco circuitos fronto-subcorticales tienen su origen en el lóbulo frontal. Además, los cinco cuentan con una proyección a las estructuras estriadas (caudado, putamen y estriado ventral) y se conectan desde el estriado hasta el globus pallidus y la sustancia negra. Desde estas dos estructuras se proyectan hacia núcleos talámicos específicos, existiendo, además, una conexión de retorno hacia el lóbulo frontal. Siguiendo la descripción de Miller y Cohen (2001), la corteza prefrontal neocortical comprende: la región lateral dorsal (áreas 46 y 8), la lateral ventral (áreas 12 y 45), la medial orbital (áreas 10, 11, 13 y 14) y la medial dorsal ( área 9). El área de apoyo, que recibe y envía información para la ejecución correcta de la función ejecutiva, es la porción anterior de la circunvolución supracallosa o paleocorteza cingulada (áreas 24 y 32); esta área se encarga de supervisar la calidad de las funciones ejecutivas y controla los mecanismos de anticipación de consecuencias y de los errores (Miller y Cohen, 2001).

Por su parte, el área 9 recibe información de la corteza visual dorsal, auditiva de la circunvolución temporal superior, somatosensorial de la parte caudal del lóbulo parietal. Éste, a su vez, recibe información de la porción superior del vermis superior y multimodal, desde la porción rostral de la circunvolución temporal superior y del núcleo dentado del cerebelo. Desde el área 9 se envía información a la corteza prefrontal (área 46), ventrolateral (áreas 12 y 45) y medial orbital (áreas 10, 11, 13 y 14). A su vez, las áreas 10, 11,12 ,13 y 14 actúan sobre la corteza motriz suplementaria y del control de la mirada (área 8), del cerebelo y del colículo superior. Las áreas 10,11, 13 y 14 actúan también sobre el hipocampo, la parte medial de la neocorteza del lóbulo temporal y, a través de ésta, sobre el tálamo y los ganglios basales, la amígdala y el hipotálamo.

El lóbulo prefrontal desempeña funciones de diferente naturaleza, incluyendo las de carácter cognitivo, emocional y de inhibición de respuestas inadecuadas. Por tanto, las disfunciones de la función ejecutiva se manifestarían en: dificultades para generar la conducta propositiva; incapacidad para resolver problemas planificándolos de forma estratégica; escasa habilidad para prestar atención a diferentes aspectos de un problema de forma simultánea; dificultad para orientar la atención y variar su enfoque de forma flexible; incapacidad para inhibir respuestas espontáneas erróneas; incapacidad para captar lo esencial de una situación compleja; baja resistencia a la distracción y control de las interferencias; incapacidad para mantener la conducta hasta lograr el objetivo y

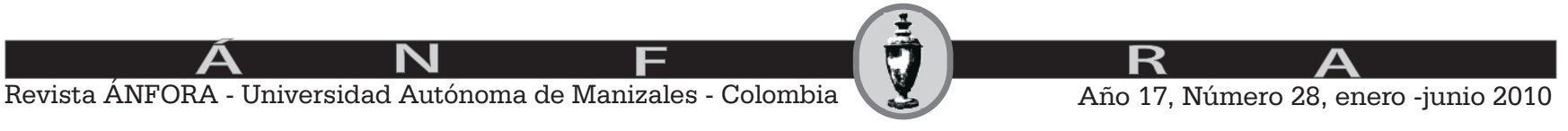


escasa habilidad para organizar y manejar el tiempo disponible para realizar las tareas.

La corteza prefrontal, por tanto, desempeña funciones muy diferentes, pero necesarias en la planificación, control e inhibición de las conductas orientadas a un propósito. Pero además, los cinco circuitos frontoestriados, involucrados en las funciones conectan con todas las zonas corticales que aportan la información sensorial necesaria para la toma de decisiones. Estos circuitos son: a) circuito esqueletomotor; b) circuito oculomotor; c) circuito dorsolateral prefrontal; d) circuito lateral orbifrontal y, e) circuito cingulado anterior.

De todos ellos, el dorsolateral prefrontal es el más involucrado en el TDAH, puesto que de él dependen fundamentalmente las funciones ejecutivas. Es evidente que, al revisar las funciones atribuidas al lóbulo frontal, se pueden establecer relaciones con diversos síntomas presentes en diferentes trastornos del desarrollo, además de estar vinculados con los del TDAH (Fuster, 1988; Pennington y Oxonoff,1996). Este hecho contribuye a explicar gran parte de los aspectos sobre comorbilidad, pero es preciso determinar una cierta especificidad en la forma en que se combinan tales disfunciones.

En este sentido, el núcleo disfuncional parece ubicarse en el déficit de inhibición de respuestas (Barckley,1995). Dicho déficit afecta a tres tipos de conductas interrelacionadas: En primer lugar, el que afecta a la inhibición de la respuesta prepotente inicial ante un evento. En segundo término, el que dificulta la detención de una respuesta ya iniciada o un patrón conductual cuasi-automatizado, impidiendo demorar la decisión o mantener la respuesta, en función de las circunstancias. Y en tercera posición, está afectado el control de la interferencia, deteriorando la protección del período de demora y las respuestas autodirigidas, que surgen como consecuencia de otras respuestas o eventos estimulantemente competitivos.

Si se carece del control necesario sobre las respuestas, es muy probable que no se puedan desarrollar correctamente las funciones ejecutivas. Éstas quedarían bloqueadas por la falta de control, impidiendo la inhibición de la conducta cuando ésta no es la adecuada. Esta tercera función ejecutiva, vinculada a la inhibición de respuestas, es lo que se denomina "acción autodirigida". Dicha acción permite el análisis y síntesis de la propia conducta, e implica la

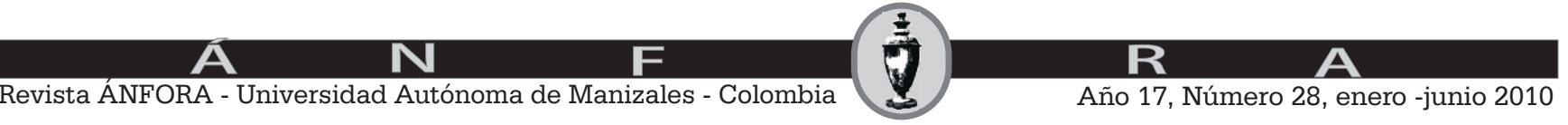


capacidad de analizar la conducta en sus unidades integrantes. Las unidades de conducta, a su vez, pueden recombinarse para crear nuevas conductas, diferentes de los patrones previamente aprendidos por el individuo.

Además, la inhibición de respuestas y, por tanto, la acción autodirigida, son responsables de la fluencia, tanto verbal como no verbal. Por ello, la fluencia motriz, que se manifiesta mediante el dibujo, la escritura y otras actividades, se ve alterada cuando el mecanismo inhibidor no funciona correctamente. Por otra parte, la autorregulación también afecta a la esfera emocional y motivacional (incluyendo la regulación del arousal, que subyace a ambos procesos). Esto implica que, en caso de que la autorregulación sea deficitaria, se verían alteradas la capacidad para dirigir la conducta, en ausencia de recompensa externa, la demora de respuestas prepotentes y el autocontrol emocional.

En conjunto, la investigación neuropsicológica del TDAH ha mostrado que la hiperactividad refleja un déficit de inhibición motriz voluntaria. Precisamente, uno de los seis subtipos establecidos por Conners y Wells (1986) se denomina "tipo disfunción del lóbulo frontal". La variedad de síntomas del TDAH abarca un amplio espectro de habilidades aparentemente muy distintas entre sí, pero cuyo denominador común es ser consideradas como funciones ejecutivas $(\mathrm{FE})$ y estar relacionadas con la disfunción de los lóbulos prefrontales del cerebro (Douglas, 1988; Fuster, 1988).

Los grupos neuronales en la corteza prefrontal alteran la frecuencia y la duración de sus descargas, antes, durante y después de resolver voluntariamente ciertos problemas. En concreto aquellos que implican una o más reglas, sin que exista un patrón uniforme para todos ellos (Stein, Xue y Belluzzi,1993). La corteza prefrontal neocortical aparece filogenéticamente con los mamíferos y está más desarrollada en los humanos que en otras especies. Su desarrollo estructural y funcional es el más tardío de toda la neocorteza y ocupa el 30\% de ella (Diamond, 2002; Gogtay, Giedd, Lusk, Hayashi, Greenstein y Vaituzis, 2004).

Vinculadas con el desarrollo de la corteza prefrontal, las funciones ejecutivas emergen como sigue: $\mathrm{El}$ inicio de las funciones ejecutivas ocurre sobre los 12 meses de edad y se desarrolla lentamente, observándose dos picos a los 4 y 18 años, para estabilizarse después. Su evolución, presenta una forma de U invertida, declinando en la vejez (Diamond, 2004). La velocidad de ejecución, la

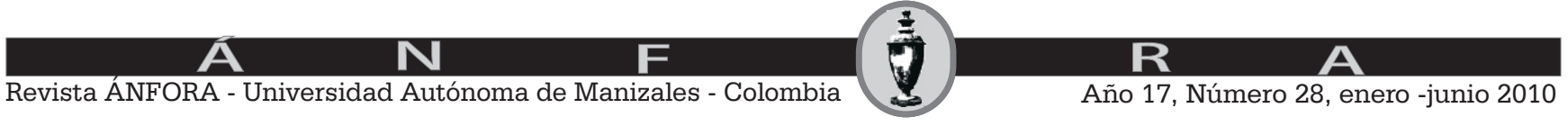


capacidad para resolver problemas complejos y la activación de áreas de la corteza prefrontal lateral dorsal y lateral ventral aumentan progresivamente con la edad, como se observa en los estudios de neuroimagen durante tareas que implican la utilización de estrategias, memoria de trabajo e inhibición (Brown, Lugar, Coalson, Miezin, Petersen y Schlagar, 2005).

\section{Neurotransmisores implicados en las funciones ejecutivas.}

Las funciones ejecutivas incluyen procesos tales como planificar, organizar, mantener la pauta de respuesta, utilizar la atención selectiva y ejercer el control inhibitorio (Fuster, 1988; Pennington y Oxonoff,1996). El déficit primario del TDAH está vinculado con las disfunciones ejecutivas y se manifiesta a través de la inflexibilidad cognitiva y la incapacidad para desarrollar un plan de acción y perseverar hasta lograr la meta. La evidencia empírica sugiere que las manifestaciones conductuales de los subtipos de TDAH con hiperactividad e impulsividad están relacionadas con una disfunción subyacente de inhibición de respuestas (Barckley, 1987; Tannock y Schachar, 1998).

A continuación se sintetizan los datos disponibles respecto a las principales funciones ejecutivas desde una perspectiva neuropsicológica, siguiendo a Papazian et al. (2006).

La función neuropsicológica de la inhibición es el proceso que permite impedir la emisión de una respuesta prepotente o detener una respuesta, una vez iniciada. Además, impide la memorización de información irrelevante, la interferencia perceptual que genera distracciones. La función inhibitoria se desarrolla progresivamente, a medida que el sujeto alcanza más edad. Esto es debido a la maduración de la corteza prefrontal (lateral dorsal y medial orbital), parte del cíngulo anterior y cuerpo estriado del tálamo (Casey, Tottenham y Fosella, 2002). La función neuropsicológica de la memoria de trabajo es el proceso, con capacidad limitada, que permite almacenar, monitorizar y manejar información necesaria para realizar una tarea (Baddeley, 1992). Presenta tres modalidades: fonológica, semántica y visoespacial. Se inicia entre los 7 y los 12 meses, mejora sustancialmente entre 4 y 8 años y alcanza su máxima funcionalidad a los 11 años.

La corteza prefrontal dorsolateral y la ventral parecen implicadas en esta función, como sugiere la activación observada en esas zonas mediante técnicas

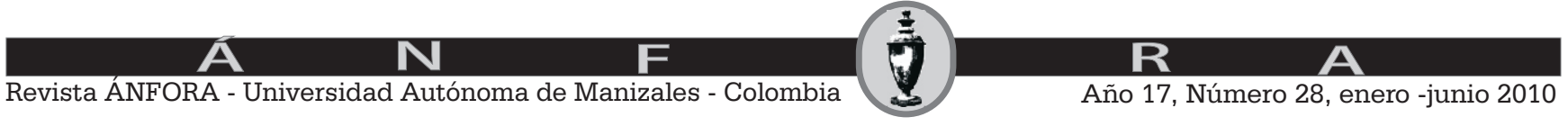


de neuroimagen funcional (Kail,1991). La función neuropsicológica del cambio de reglas permite cambiar intermitentemente de una a otras reglas de acción, imponiendo demandas adicionales a los procesos de inhibición y a la memoria de trabajo (Diamond, 2002). Esta función comienza a manifestarse a los 4 años y mejora de forma progresiva con la edad. La función neuropsicológica de la planificación de la respuesta, asociada a una respuesta, en tareas de solución de problemas es el proceso mental denominado planificación. Igual que el cambio de reglas, la planificación de respuesta impone demandas adicionales a los procesos de inhibición y a la memoria de trabajo. Su sustrato anatómico es la corteza prefrontal dorsolateral (Cabeza y Nyberg, 2000). Se manifiesta a partir de los 4 años y alcanza su máximo desarrollo a los 15. La función neuropsicológica de la monitorización y el control, proceso, también denominado metacognitivo, permite autoevaluar y controlar el proceso de planificación, previo a la toma de decisiones, con objeto de garantizar la mejor solución al problema. Esta función es dependiente de la edad, emerge a los 4 años, llega a su máxima funcionalidad en la adolescencia y tiene capacidad limitada. La función neuropsicológica de las decisiones implica, entre otras estructuras, la participación de la corteza prefrontal ventromedial (Bechara, Damasio y Damasio, 2000). Implica manejar información sobre la naturaleza de la decisión, los tipos de error cometidos previamente en decisiones similares y los riesgos que conlleva. Es un proceso mental, dependiente de la edad y con capacidad limitada. En el caso de decisiones arriesgadas, sin anticipación de consecuencias, se manifiesta entre los 6 y 12 años, mejorando de forma progresiva hasta los 25 (Crone y Van der Molen, 2004).

Para comprender, tanto el funcionamiento normal como las alteraciones de las funciones ejecutivas, es necesario conocer, al menos esquemáticamente, el papel que desempeñan los neurotransmisores implicados en las actividades ejecutivas.

Los resultados de la evaluación clínica, neuropsicológica y neuroimaginológica (Shallice y Burguess, 1991; Hughes, Russell, y Robbins, 2004), tanto en humanos como en animales, bajo la acción de agonistas y antagonistas de los neurotransmisores cerebrales, han mostrado que la corteza prefrontal es esencial para el desempeño correcto de las funciones ejecutivas y que la dopamina y la norepinefrina son los neurotransmisores más implicados (Robbins, 2001; Miller y Cohen, 2001). En el encéfalo existen varias vías

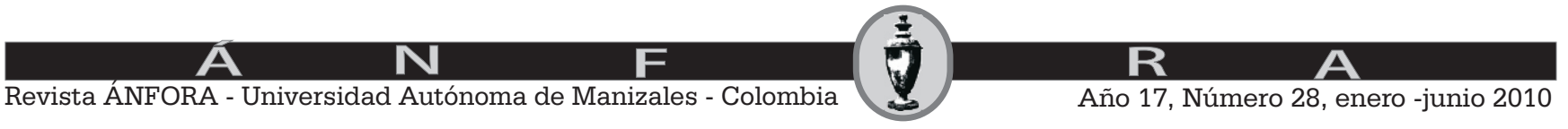


dopaminérgicas: la vía nigroestriada. Se origina en las neuronas dopaminérgicas de la sustancia negra y se proyecta hacia los ganglios basales (núcleos caudado y putamen). Forma parte del sistema extrapiramidal e influye en el control del movimiento. La hiperactividad, propia del TDAH, está relacionada con una disfunción de esta vía; la vía mesolímbica. Se origina en neuronas secretoras de dopamina de la región ventral del tegmento mesoencefálico que finalizan estableciendo sinapsis en el núcleo accumbens. La frecuencia y descarga de estas neuronas aumentan en respuesta a una recompensa inesperada y disminuyen cuando se omite dicha recompensa (Schultz, 1998). Esta vía forma parte del sistema límbico y está involucrada en muchas conductas, sensaciones placenteras etc. La necesidad de gratificación inmediata, propia del TDAH, se relaciona con la disfunción dopaminérgica mesolímbica; la vía mesocortical. Tiene su origen en la región ventral del tegmento mesoencefálico. Proyecta sobre la corteza parietal, temporal y prefrontal de asociación. Sus neuronas están implicadas, tanto en las funciones ejecutivas, como en la inhibición de la respuesta, el control motor, la atención y la memoria de trabajo (Goldman-Rakic, 1996). La disminución de la dopamina sináptica en la corteza prefrontal da lugar a trastornos en las funciones ejecutivas, especialmente en las encargadas de la planificación de la respuesta (Sagvolden, Johansen, Aase, y Russell, 2005) y la vía tuberoinfundibular que se proyecta al hipotálamo y glándula pituitaria y controla la secreción de prolactina. No parece guardar relación con el TDAH.

Como puede apreciarse, la dopamina está implicada en distintas vías, cada una de las cuales, tiene su propia función. Aunque desde el punto de vista de su funcionalidad estos sistemas se relacionan con patologías como la esquizofrenia, las funciones motrices, los estados emocionales y la memoria, las manifestaciones derivadas de la disfunción de cada una de las vías no son específicas.

En cuanto a la noradrenalina, se encuentran las siguientes vías noragrenérgicas:

Desde el locus coeruleus al córtex prefrontal, responsable de la regulación del estado de ánimo. Otras proyecciones del locus coerelus al córtex frontal, responsables de la atención, memoria de trabajo y velocidad de procesamiento de la información. Proyecciones del locus coerelus al córtex límbico, que afectan

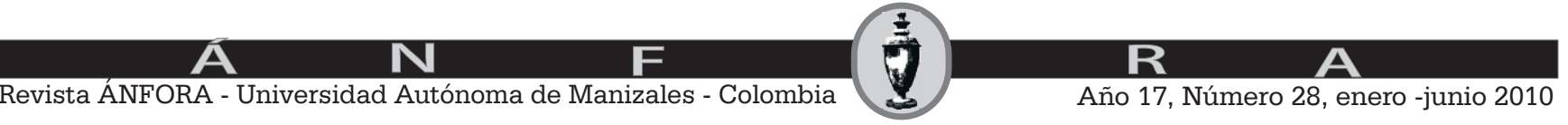


a las emociones, energía, fatiga, agitación psicomotriz y capacidad intelectual. Proyecciones del locus coerelus al cerebelo, relacionadas con ciertos movimientos, como el temblor. El síndrome de deficiencia a la noradrelina involucra la disfunción de diferentes vías noradrenérgicas. Este síndrome incluye déficit de atención, problemas de concentración, déficit en memoria de trabajo, lentitud en el procesamiento de la información, depresión, retraso psicomotor y fatiga (Artigás Pallarés, 2004).

\section{LA MOTRICIDAD Y EL TDAH.}

El potencial valor terapéutico de la motricidad en el tratamiento del TDAH, ha sido objeto de diferentes estudios en los últimos años. En la mayor parte de ellos, se contempla dentro de un tratamiento multimodal, lo que dificulta la valoración de los efectos del ejercicio de forma aislada. En otros casos, se evalúa el efecto de la motricidad (ejercicio físico y otros) sobre diversas manifestaciones del síndrome.

Así, por ejemplo, Lemura, Von Duvillard y Mookerjee (2000) han mostrado que la práctica de la motricidad sobre el funcionamiento cognitivo y, especialmente, que la actividad física vigorosa puede beneficiar a niños que padecen el trastorno por déficit de atención e hiperactividad.

Tantillo, Keisck, Hynd y Dishman (2002) publicaron un estudio donde aplicaron ejercicio aeróbico (caminata en banda rodante) en niños con el TDAH y encontraron que el ejercicio podía tener un efecto positivo en las conductas típicas del trastorno, pudiendo aportar al niño con TDAH, los siguientes beneficios: A nivel físico: corregir problemas de lateralidad o de coordinación y trabajar la motricidad gruesa y fina. A nivel educativo: favorecer el aprendizaje motor, facilitar el conocimiento del esquema corporal y propiciar la comprensión del movimiento. A nivel social: compartir una actividad de grupo, aprender a respetar normas, a aceptar a los demás y ser respetado por el grupo.

Sin embargo, este trabajo no especifica los resultados obtenidos tras el programa de ejercicio aeróbico, limitándose a proponer los supuestos beneficios que de dicho programa podrían derivarse. Alguno de tales beneficios, como por ejemplo los que los autores proponen a nivel social, serían difíciles de obtener mediante la caminata en banda rodante, ya que esta

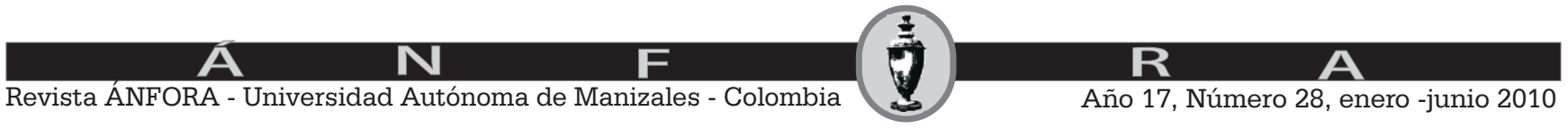


actividad no implica, en principio, la relación social ni el desarrollo del respeto propio y ajeno. Tampoco es fácil entender cómo podrían corregirse problemas de lateralidad o coordinación, ni mejorar la motricidad fina con el trabajo en la banda rodante, etc. En suma, estudios de esta índole, lejos de clarificar las relaciones entre el ejercicio físico y el TDAH, pueden, incluso, inducir a confusión.

En relación al ejercicio, como parte del tratamiento, Barkley (2002) presenta estudios de caso de los pacientes con TDAH en cuyo tratamiento se incluye el ejercicio físico y/o la práctica deportiva, fundamentalmente de carácter aeróbico intenso, afirmando haberse observado un claro beneficio. En ninguno de estos estudios se operativiza el tipo de ejercicio prescrito a los sujetos, ni se cuantifican las mejoras obtenidas. Lo que ofrece el autor es una descripción somera del tratamiento, indicando la prescripción de ejercicio y se añade un comentario anecdótico sobre los efectos obtenidos.

Winnick (2004 p.153) estudió 19 niños con TDAH que tomaban estimulantes y constató que el desempeño de estos niños, en términos de aptitud física y habilidades motrices gruesas, estaba por debajo de la media, comparada con los estándares establecidos para los niños de estas edades y género. También mostraron como la incompetencia atlética y el fracaso escolar podían contribuir para que estos niños mantuvieran un autoconcepto bajo, lo que les ocasionaba un sentimiento de desvalorización y frustración frente a actividades de relaciones sociales.

Vidarte, Ezquerro y Giraldez (2009), muestran como el perfil psicomotor de los niños diagnosticados con TDAH en la ciudad de Manizales Colombia, es eupráxico y se clasifica en la misma categoría que el de los niños sanos, aunque su puntuación es menor y los niveles de ejecución en los factores, tonicidad, equilibrio, lateralidad, noción de cuerpo, estructuración espacio temporal, Praxia global y Praxia fina son peores. De modo excepcional, no se encuentran diferencias en la lateralidad, pero en este caso es igualmente deficitaria en los niños sanos. Al comparar los factores psicomotores de los niños con TDAH y sanos en las diferentes edades, se aprecia que en ambos grupos mejoran con los años, aunque los niños con TDAH siempre muestran un retraso respecto a los sanos.

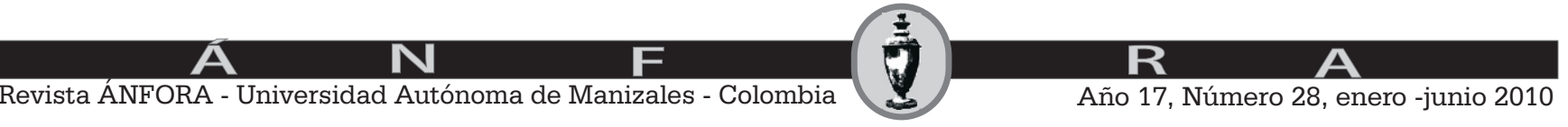


La motricidad puede proporcionar un campo grande de aprendizaje para mejorar las habilidades sociales en los sujetos con TDAH. Si existen diferentes causas y formas de TDAH, debe haber también varias formas de enseñar a los niños con este trastorno. Winnick (2004 p.156) sugiere estrategias específicas de enseñanza para profesores que ayuden a los niños con TDAH a mantener la atención y concentración en los diferentes ambientes escolares. Estas estrategias implican el abordaje de rutinas altamente estructuradas y consistentes, el establecimiento de reglas, el uso de programas de control del comportamiento, expresar claramente todas las expectativas esperadas, escoger actividades que involucren movimientos lentos y controlados para disminuir la hiperactividad e impulsividad, destacar comandos relevantes, estimular permanentemente al niño, variar permanentemente las tareas motrices, minimizar las actividades de juegos competitivos y estimular la cooperación.

En la misma línea, Tantillo et al (2002) recomiendan la actividad física a los niños con TDAH, hasta donde sea posible. Consideran que se les debe proveer por lo menos una hora diaria de aeróbicos, karate do, natación, danzas, gimnasia u otros deportes. Estos autores sostienen que el gasto energético realizado por el niño al hacer ejercicio le permitirá mayores niveles de quietud. Además, argumentan que el ejercicio superior al nivel la condición física requiere mayor concentración del niño, lo que se generalizaría a tareas de índole intelectual. Aunque ambos argumentos puedan ser razonables, disminuir la hiperactividad mediante el aumento del gasto energético lograría, en todo caso una reducción temporal y no una modificación de los mecanismos neuropsicológicos que subyacen en la etiología del trastorno. Asimismo, esperar una transferencia desde la supuesta concentración generada por el ejercicio físico hacia las tareas intelectuales sugiere un conocimiento muy superficial del funcionamiento de los procesos atencionales.

En general, los estudios sobre la influencia del ejercicio físico en los síntomas del TDAH son excesivamente simplistas y lastrados por deficiencias metodológicas. Esto dificulta conocer con precisión: el efecto del ejercicio físico sobre síntomas concretos del TDAH, el tipo de ejercicio más adecuado en la mejora de tales síntomas, las vías neurológicas y bioquímicas mediante las cuales se expliquen las mejoras obtenidas y la eficacia del ejercicio frente a otros tratamientos tradicionales, o como parte del tratamiento multimodal.

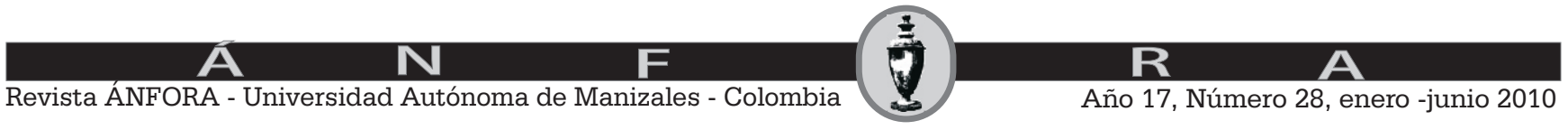


A manera de conclusión se puede plantear que es necesario propiciar que las personas aprecien y entiendan la dimensión corpórea de su existencia, en donde se procure lograr que las experiencias que les son ofrecidas no se transformen en simples catálogos de actividades desprovistas de significado. En este proceso se deben tener presentes también las posibles alteraciones del desarrollo psicomotor del sujeto, por tanto la motricidad por la que apostamos es la de la estimulación de la afectividad positiva, de reto a la cognición, de encuentro con los demás, de aceptación reconciliadora con su cuerpo en movimiento, de invitación a dar lo mejor que podemos, de búsqueda de nuestra sonrisa, de curiosidad por lo que puede ser, de, sencillamente: Ser Humano.

\section{Bibliografía}

PARLEBAS, J.P. 2007. Juego, deporte y sociedad. Léxico de praxeología motriz. Edición 1. Paidotribo. Barcelona España

TRIGO, E. y COL. (2.000). Creatividad y motricidad. Madrid: Inde.

MURCIA, N. (2006). Juan Luís Pintos: las relevancias y opacidades en los imaginarios sociales. En: Vida universitaria. Un estudio desde los imaginarios de maestros y estudiantes. Tesis doctoral. (pp.43-52). Manizales: CINDE Universidad de Manizales.

MURCIA, P. NAPOLEÓN OSPINA Héctor Fabio. (2007). Motricidad Humana y globalización. En: Revista Hologramática, facultad de ciencias sociales, UNLZ, año 4 No. 6. (2007) 3-23.Buenos Aires.

HURTADO, D.R. (2007) Corporeidad y motricidad, una forma de mirar los saberes del cuerpo. Educ. Soc., Campinas, vol. 29, n. 102, p. 119-136, jan./abr. 2008, Disponível em < http://www.cedes.unicamp.br $>$

FEITOSA, A. M. Contribuciones de Tomas Kuhn para una epistemología de la motricidad humana. Lisboa. Instituto Piaget. 1993.

REY, A. y CANALES, I. 2007. Discurso Epistémico para una Ciencia de la Motricidad Cinta de Moebio 28: 104-123 www.moebio.uchile.cl/28/rey.html

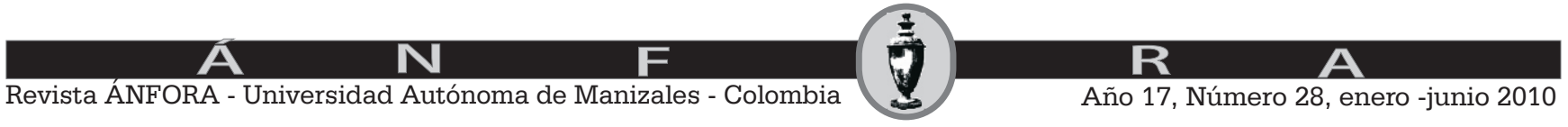


TRIGO, E. y COL. (1999). Creatividad y motricidad. Madrid: Inde.

DA FONSECA (1989): Desenvolvimiento humano. Da filogénese á ontogénese da motricidade. Lisboa: Noticias.

WALLON, H. (1970). Les Origines du caractére Chez lEnfant, PUF, París.

LURIA, A. R. (1985). Consciencia y lenguaje. Pablo del Río, Madrid.

MAZET, P y HOUZAL , A. (1981) Le Sommeil de L'Enfant et ses Troubles. Presses. Universitaires de France, Iré édition, 127 Págs.

NEISSER, U. (1976) Psicología Cognoscitiva. Trillas.

PIEK, PITCHER y HAY. (1999). Motor coordination and kinaesthesis in boys with attention deficit-hyperactivity disorder. School of Psychology, Curtin University of Technology, Perth, Western Australia, Australia.

STEGER, J., IMHOF, K., GUNDELFINGER, R., STEINHAUSEN, HC., y BRANDEIS, D. (2001). Attentional and neuromotor deficits in ADHD. Dev Med Cild Neurol; 43: 172-9

LEUNG PWL, CONNOLLY KJ. (1998). Do hyperactive children have motor organization and/or execution deficits? Dev Med Child Neurol 1998; 40: 600-7. 10. Rutter M. Syndromes attributed to 'minimal brain dysfunction' in childhood. Am J Psychiatry 1982; 139: 21-33

CASTROVIEJO, P. (2004). Síndrome de déficit de atención con hiperactividad y capacidad para el deporte Revista de Neurología. Rev, Neurol; 38 (11): 1001-1005

BARKLEY, R. A., DUPAUL, G. J. y MCMURRAY, M. B. (1990). Comprehensive evaluation of attention deficit disorder with and without hyperactivity as defined by research criteria. Journal of Consulting and Clinical Psychology, 58, 6, 775-789.

KADESJO, B. y GILBERT, C. (1988). Attention deficit and clumsiness in Swedish 7 year old children. Dev Med Chil Neurol 40: 796-804.

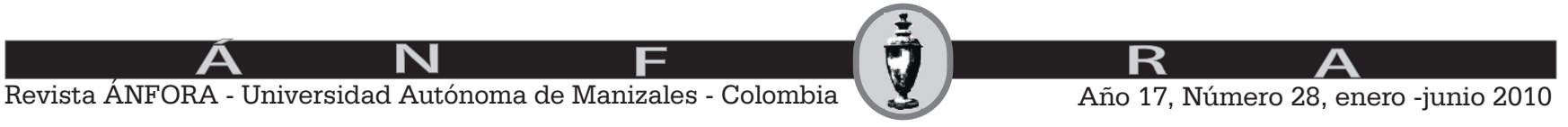


DOYLE, S., WALLEN, M. y WHITMONT, S. (1995). Motor Skills in Australian children with attention deficit hyperactivity disorder. Occup Ther Int; 2: 229-40.

ORJALES I. (2002) Programa de intervención cognitivo-conductual para niños con déficit de atención con hiperactividad. Madrid: CEPE.

BAUERMEISTER, J.J. (2002). Hiperactivo, Impulsivo, Distraído, ¿Me conoces?. Guía acerca del déficit atencional para padres, maestros y profesionales. Guilford: New York

YOCHMAN, ORNOY y PARUSH (2006) Co-occurrence of developmental delays among preschool children with attention-deficit-hyperactivity disorder. Developmental Medicine \& Child Neurology 48 (6), 483-488.

LICARI, LARKIN y MIYAHARA (2006). The influence of developmental coordination disorder and attention deficits on associated movements in children. Feb, 25 (1):90-9 Epub

COLBY, C. L. (1991). The neuroanatomy and neurophysiology of attention. Journal of Child Neurology, 16, S90-S118

CASTELLANOS, F. X., GIEDD, J. N., ECKBURG, P., MARSH, W. L., VAITUZIS, C., KAYSEN, D., HAMBURGER, S. D. y RAPOPORT, J. L. (1994). Quantitative morphology of the caudate nucleus in attention deficit hyperactivity disorder. Neurology, 151, 12, 1791-1796.

CASTELLANOS, F. X., GIEDD, J. N., HAMBURGER, S. D, MARSH, W. L. y RAPOPORT, J. L. (1996a). Brain morphometry in Tourette syndrome, the influence of comorbid attention-deficit/hyperactivity disorder. Neurology, 47, 1581-1583.

BARKLEY, R. A., GRODZINSKY, G. y DUPAUL, G. J. (1992). Frontal lobe functions in attention deficit disorder with and without hyperactivity: A review and research report. Journal of Abnormal Child Psychology, 20, 163- 188

MILLER, EK. y COHEN J. D. (2001). An integrative theory of prefrontal cortex function. Annu Rev Neurosci; 24: 167- 202.Milich, R., Hartung, C. M., Matin C. A.,

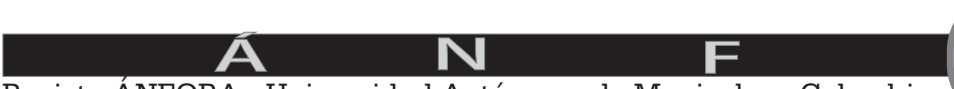


y Haigler, E. D. (1995) Behavorial disinhibition and underlying processes in adolescents with disruptive behavior disorders. In Routh DK, ed. Disruptive behavior disorders in child-hood. New York: Plenum Press

FUSTER JM. (1988). The prefrontal cortex: Anatomy, phisiology and neuropsichology of the frontal lobe (2 on ed). New York: Raven; 1989.

PENNINGTON B. F. y OXONOFF, S. (1996). Executive functions and developmental psychopathology. J. Child Psychol Psychiatry; 37: 51-87.

BARKLEY, R. A. (1995). Attention deficit hyperactivity disorder: A handbook for diagnosis and treatment. Nueva York: Guilford Press.

CONNERS CK. y WELLS, KC. (1986). Hiperkinetic children: a neuropsychosocial approach. Beverly Hills; Sage.

DOUGLAS V. I. (1988). Cognitive deficits in children with attention deficit disorder with hyperactivity. En: Bloomingdale LM., Sergeeant J.,( eds.) Attention deficit disorder: criteria, cognition, intervention. New York; Pergamon Press.

STEIN, L., XUE, B.G. y BELLUZZI, J. D. (1993). A cellular analogue of operant conditioning. Journal of the Experimental Analysis of Behavior, 60, 41-53.

GOGTAY, N., GIEED, J., LUSK, L., HAYASHI K., GREESTEIN D. y VAITUZIS, A. (2004). Dynamic mapping of human cortical development during child-hood through early adulthood. Proc Na.

DIAMOND, A. (2002). Normal development of prefrontal cortex from birt to young adulthood; cognitive function, anatomy, and biochemistry. In Struss DT, Knigth RT. Eds. Principles of frontal lobe function London; Oxford University Press; p. 466-503.

DIAMOND, A. (2004) La magnetoncefalografía para el diagnostico pedagógico del TDAH. En Rev. Neurol; 39: 183.

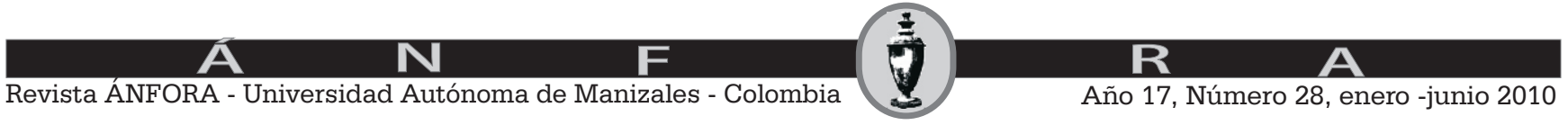


BROWWN,T., LUGAR H., COALSON, R., MIEZIN, F., y PETERSEN, S., (2005). Develpometal changes in human cerebral functional organization for word generation. Cereb Cortex; 15:275-90.

PENNINGTON B. F., Y OXONOFF, S. (1996). Executive functions and developmental psychopathology. J. Child Psychol Psychiatry; 37: 51-87.

BARKLEY, R. A. (1997). ADHD and the nature of self-control. Nueva York: Guilford Press

TANNOCK, R. y SCHACHAR R. (1998) Executive dysfunction as an underlying mechanism of behavior and language problems in attention deficit hyperactivity dosroder. En: Beitchman JH, Cohen NJ, (EDS.)Language, learning and behavior disorders; developmental biological, and clinical perspectives. New York: Cambridge University Press. P. 128-55.

TANTILLO, KEISCK, HYND y DISHMAN (2002). Physical education and behaviour.Exploring the relationship between physical education and enhancing behaviour in pupils with emotional behavioural difficulties. Support for Learning 21 (4), 169-174.

PAPAZIAN, O., ALFONSO I. y LUZONDO, R. (2006) Trastornos de las funciones ejecutivas. Simposio de trastornos del desarrollo. Rev Neurol: 42 /supl 3): S45S50.

BADELEY A., (1992). Working memory. Science. 255: 556-9.

CASEY, B. J. TOTTENHAMN, N. y FOSELLA, J. (2002). Imaging, Lesion and genetic approaches toward a model of cognitive control. Dev Psychobiol; 40: 237-54.

KAIL, R. (1991). Developmental change in speed of processing during childhood and adolescence. Physichol Bull: 109: 490-501.

CABEZA, R. y NYBERT, L. (2000). Imaging cognition II: and empirical review of 275 PET and FMRI studies. J Cogn Neurosci; 12: 1-47.

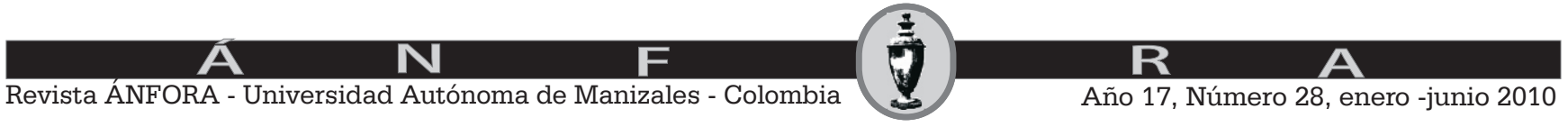


BECHARA A., DAMASIO, H. y DAMASIO, A. R. (2000). Emotion, decision making and the orbitofrontal cortex.Cerb Cortex; 10: 295-307.

CRONE, E. A. y VAN DER MOLEN, M. (2004). Development changes in real life decisión parking: performance on a gambling task previusly shown to depend on the ventromedial prefrontal cortex. Dev Neuropsychol; 25: 251-79.

SHALLICE, P. W. y BURGESS, P. W. (1991) Deficits in strategy application following frontal lobe damage in man. Brain 114, 727-741.

HUGHES, RUSSELL y ROBBINS: (2004). Evidence for executive dysfunction, ReV Neuropsychol; 32: 477-92.

ROBBINS, W. (2001). Chemical neuromodulation of frontal-executive functions in humans and other animals. Exp Brain Res; 133: 130-8.

MILLER, EK. y COHEN J. D. (2001). An integrative theory of prefrontal cortex function. Annu Rev Neurosci; 24: 167- 202.Milich, R., Hartung, C. M., Matin C. A. y Haigler, E. D. (1995) Behavorial disinhibition and underlying processes in adolescents with disruptive behavior disorders. In Routh DK, ed. Disruptive behavior disorders in child-hood. New York: Plenum Press.

SCHULTZ, J.H. (1998). IAnatomie daos la Psychanalyse in Nouvelle Revue de Psychanalyse Lieux du Corps. Gallimar, París.

GOLDMAN, R. Y RAKIC, P. S. (1998). Tomography of cognition: parallel distributed networks in primate association cortex. Annuary Review of neuroscience, 11, 137-156.

SAGVOLDEN, JOHANSEN, AASE, Y RUSSELL, 2005). A dynamic developmental theory of attention-deficit/hyperactivity disorder (ADHD) predominantly hyperactive/impulsive and combined subtypes. Department of Physiology, University of Oslo, NO-0317 Oslo, Norway.

ARTIGAS-PALLARÉS, J. (2004). Nuevas opciones terapéuticas en el tratamiento del trastorno por déficit de atención/ hiperactividad. Rev Neurol; 38 (supl 1):S117-S123.

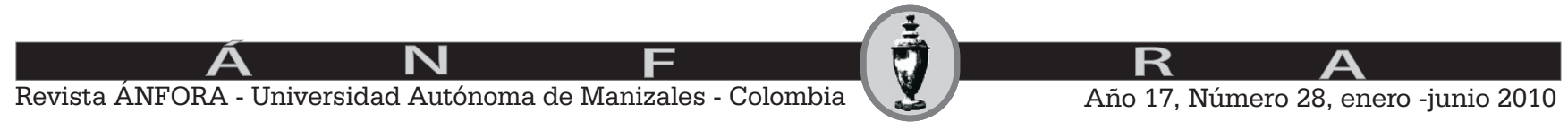


LEMURA, VON DUVILLARD y MOOKERJEE (2000). The effects the physical training of functional capacity in adults, ages 66 to 90: A meta- analysis. Journal of sport Medicina and Physical Fitness 40 (1) 1-10

WINNICK, J. P. (2004). Educação Física e Esportes Adaptados. Barueri: Manole.

BARKLEY, R. A.(2002). Excessive dopamine transporter density in adults with attention deficit hyperactivity disorder assessed by SPECT with [123 I] altropane. University of Massachusetts Medical School,Worcester, MA.

VIDARTE, J.A., EZQUERRO, M. GIRALDEZ, MA (2009) Perfil psicomotor de los niños entre 5 y 12 años diagnosticados clínicamente con TDAH en la ciudad de Manizales, Colombia. Revista de Neurología. Rev. Neurol: ;49 (2): 69-75 